ful counting shows that $B$ is empty.) The seven-person game, $k=2$, is well known.

Added in proof. The 15 solutions mentioned at the end of $\S 3$ are main simple, with $x_{7}=0$. The projective plane games and their solutions $V_{k+1}$ were previously described in an unpublished paper of Moses Richardson.

The George Washington University

\title{
CERTAIN TYPES OF HOMOGENEOUS CONTINUA
}

\author{
C. E. BURGESS
}

According to the usual definition of homogeneity, a point set $M$ is said to be homogeneous if for any two points $x$ and $y$ of $M$ there is a homeomorphism of $M$ onto itself carrying $x$ into $y$. Some more general types of homogeneity previously defined in [2] will be studied in this paper, and it will be shown that there is a certain type of homogeneity such that every decomposable compact metric continuum possessing it is a simple closed curve. For bounded plane continua possessing the usual type of homogeneity, this problem has been only partially solved. ${ }^{1}$ Added in proof. At the Summer Meeting in Laramie, September, 1954, Bing and Jones each presented an example of a decomposable homogeneous bounded plane continuum which is different from a simple closed curve [Bull. Amer. Math. Soc. Abstracts 60-6-766 and 60-6-770].

THEOREM 1. If every proper subcontinuum of the compact metric continuum $M$ is nearly homogeneous, then $M$ is hereditarily indecomposable. $^{2}$

Proof. Since every subcontinuum of $M$ satisfies the hypothesis of this theorem, it will be sufficient to show that $M$ is indecomposable.

Suppose that $M$ is decomposable. Then there is some proper sub-

Presented to the Society, May 1, 1954; received by the editors May 5, 1954 and, in revised form, August 2, 1954. in $[2]$.

${ }^{1}$ For these results, see [3, Theorem 2], [2, Theorem 8], and other references cited [1].

${ }^{2}$ An example satisfying the hypothesis of this theorem has been described by Bing 
continuum $H$ of $M$ such that the closure of $M-H$ does not contain $H$. Let $x$ be a point of $M-H$, and let $K$ be a subcontinuum of $M$ irreducible from $x$ to $H$. Since $K-K \cdot H$ is a subset of $M-H$ and $K$ is the closure of $K-K \cdot H$, it follows that $K$ does not contain $H$. Let $y$ be a point of $H-H \cdot K$, and let $H^{\prime}$ be a subcontinuum of $H$ irreducible from $y$ to $K$. Since $K$ is nearly homogeneous and is irreducible between some two points, it follows from [2, Theorem 4] that $K$ is indecomposable. Hence some composant of $K$ intersects both $H^{\prime}$ and $K-K \cdot H^{\prime}$, and this composant contains a continuum $K^{\prime}$ irreducible from $H^{\prime}$ to some point $z$ of $K-K \cdot H^{\prime}$. Then $H^{\prime}+K^{\prime}$ is a proper subcontinuum of $M$ and is irreducible between $y$ and $z$. Hence $H^{\prime}+K^{\prime}$ is nearly homogeneous, and by [2, Theorem 4], it is indecomposable. This involves a contradiction since both $H^{\prime}$ and $K^{\prime}$ are proper subcontinua of $H^{\prime}+K^{\prime}$. Hence $M$ is indecomposable.

Theorem 2. If $M$ is a decomposable compact metric continuum such that for every two nondegenerate proper subcontinua $H$ and $K$ of $M$ there is a homeomorphism of $M$ onto itself that carries $H$ onto $K$, then $M$ is a simple closed curve. ${ }^{3}$

Proof. Since $M$ is decomposable, then some proper subcontinuum of $M$ is not a continuum of condensation ${ }^{4}$ of $M$. Hence no nondegenerate proper subcontinuum of $M$ is a continuum of condensation of $M$. This implies that $M$ is locally connected. Clearly $M$ is not an arc, and since every nondegenerate locally connected compact continuum which is neither an arc nor a simple closed curve contains both an arc and a simple triod as proper subsets [5, p. 446], it follows that $M$ is a simple closed curve.

Theorem 3. If $n>1$ and the plane continuum $M$ is nearly $n$-homogeneous and is not locally connected, then $M$ is indecomposable.

A proof for the case in which $M$ is bounded was indicated in [2, Theorem 9]. If $M$ is unbounded, the method of inversion can be applied so that the proof is quite similar to the one for the bounded case.

THEOREM 4. If $n>1$ and the unbounded continuum $M$ is n-homogeneous and is a proper subset of the plane, then $M$ is homeomorphic with a straight line.

\footnotetext{
${ }^{3}$ Bing [1, Theorem 15] has shown that the requirement that $M$ be decomposable is necessary.

A proper subcontinuum $K$ of $M$ is said to be a continuum of condensation of $M$ if every point of $K$ is a limit point of $M-K$.
} 
Proof. The argument used in the proof of [2, Theorem 10] can be used to show that $M$ is decomposable. Hence by Theorem $3, M$ is locally connected. By [2, Theorem 1], $M$ is homogeneous. Mazurkiewicz $[4$, p. 146] has shown that every locally connected unbounded homogeneous proper subcontinuum of the plane is homeomorphic with a straight line.

\section{BIBLIOGRAPHY}

1. R. H. Bing, A homogeneous indecomposable plane continium, Duke Math. J. vol. 15 (1948) pp. 729-742.

2. C. E. Burgess, Some theorems on n-homogeneous continua, Proc. Amer. Math. Soc. vol. 5 (1954) pp. 136-143.

3. F. Burton Jones, Certain homogeneous unicoherent indecomposable continua, Proc. Amer. Math. Soc. vol. 2 (1951) pp. 855-859.

4. Stefan Mazurkiewicz, Sur les continus homogènes, Fund. Math. vol. 5 (1924) pp. 137-146.

5. R. H. Sorgenfrey, Concerning triodic continua, Amer. J. Math. vol. 66 (1944) pp. $439-460$.

UNIVERSITY OF UTAH 